\title{
VARICOCELE, NAEUS, AND VARICOSE VEINS OF THE LEG TREATED BY THE METHOD OF SUTURE.
}

\author{
Br CHARLES B. BALL, M.Cr., F.R C.S.; \\ Surgeon to Sir Patrick Dun's Hospital.
}

[Read in the Surgical Section, March 15, 1895.]

The arrest of bleeding by stitching the cut surfaces together dates back to a very remote period of surgical history. The introduction of the more direct methods, however, such as ligature, \&c., appears so completely to have usurped the surgical mind, that but little was heard for a long time of the introduction of sutures solely for the suppression of hæmorrhage. One notable exception, however, exists. In wounds of the lips, either from injury, or from the operations for hare-lip and epithelioma, the universal practice has been to rely on the sutures for the arrest of bleeding from the coronary arteries; but in almost all other cases reliance was placed on ligatures applied directly to the arteries, and sutures were only used for the purpose of coaptation of the edges of the wound. Quite insensibly, however, the progress of modern surgery has shown how much can be done to dispense with the direct ligation of vessels of moderate size and substitute a system of deep sutures for the arrest of bleeding. It appears astonishing that with the good results universally obtained in wounds of the lip so constantly before us, we should have been so long in adapting this principle of treatment to other cases. The contrast between an excision of the breast twenty-five years ago and to-day illustrates very clearly the advantages of this method. Formerly, when a cancer of the breast was removed, all bleeding points were ligatured (often 
ten or twelve), the wound was brought together with a few points of interrupted suture passed only through the skin, and very explicit instructions were given to avoid anything like tension on the sides of the wound. The result was the formation of an extensive blood-clot in the wound, a copious blood-staining of the dressings; while severe intermediary hæmorrhage was not infrequent. Now, by pressure-forceps, bleeding is temporarily arrested, and, subsequently, a series of deep sutures, so as effectually to close the wound cavity, permanently arrests further hæmorrhage. In my own practice I rarely ever apply any ligature whatever in excision of the breast; four or five deep sutures are passed entirely under the wound cavity, and, before closing these, the edges are brought accurately together by a fine continuous suture, no drainage-tube being used; the deep sutures are now firmly closed over lead plates and, usually, bleeding is absolutely stopped; if hæmorrhage still continues from any point, an additional deep suture will usually make the wound absolutely dry. The result is that there is, practically, no bloody serum discharged from the wound, and the original dressings can be left undisturbed for many days. In amputations, also, only the very largest arteries are ligatured, complete coaptation of the surfaces effectually stopping all minor bleeding. In Whitehead's operation for piles, reliance is altogether placed on sutures for the arrest of hæmorrhage from the very vascular surface left by the excision of the "pile area," with the result that, if properly done, after-bleeding occurs much less frequently than after the other operations; indeed, it is quite usual to find that the pad of dressing applied to the anus is scarcely blood-stained after twenty-four hours. What, then, are the manifest advantages of this system over the plan of applying ligatures to every bleeding point? Decreased hæmorrhage from weeping of the cut surfaces; no wound cavities which require to be drained, and in which 
blood-clots can accumulate; and no ligatured ends of vessels to necrose, or foreign bodies in the shape of ligatures. In a perfectly aseptic wound no doubt a limited quantity of necrotic tissue, blood-clot, or ligature material can be absorbed; but the difficulty of maintaining asepsis may be said to increase directly with the quantity of such material in the wound. The less there is, the more certain and rapid is the healing.

This system of wound treatment appears to be capable of considerable development, and suggests many problems of much interest to discuss. I propose to consider three operations in which it appears to me an extension of this method constitutes a very considerable improvement in the operative detail. They are : varicocele, nævus, and varicose veins in the leg.

I take it as admitted that the subcutaneous operations for varicocele are obsolete, and that the only one now claiming attention is the excision of the mass of veins. Upon exposing the spermatic cord in an extensive case of varicocele, the veins forming the pampiniform plexus are found widely dilated and tolerably adherent together, so that, although difficult to separate from one another, they can easily be isolated en bloc from the vas deferens. We were formerly told that the spermatic artery could readily be separated with the vas from the mass of veins. Mr. Treves has, however, shown that the spermatic artery is more closely related to the mass of veins than it is to the vas deferens, so that, in all probability, in the majority of cases of this operation, the spermatic artery suffers occlusion. This, however, does not appear to be a matter of much importance as the deferential artery, which closely adheres to the vas, inosculates freely with the branches of the spermatic artery over the epididymis, and is quite sufficient to maintain the circulation. There can also be seen a plexus of minute veins accompanying the deferential artery and surrounding the vas, which are 
not dilated, and which belong to a system quite separate from the spermatic. This deferential vascular system appears. to be quite competent to completely carry on the testicular circulation when the spermatic artery and veins are thoroughly excised; and it is obvious that by thus transferring the circulation to another and undiseased system, complete and permanent cure is probable. A single case of sloughing of the testis is recorded, but, as. it is an isolated example, it does not carry much weight. The way in which $\mathrm{I}$ now operate is as follows: An incision $1 \frac{1}{2}$ inches long is made through all the covering of the cord, completely exposing the plexus of veins. The vas deferens is now carefully separated from the mass of veins for the whole length of the cord from close to the external abdominal ring down to the epididymis. This can readily be done, through the small incision made, by drawing the elongated cord out in a loop. A clamp forceps is now placed on the mass of veins at the

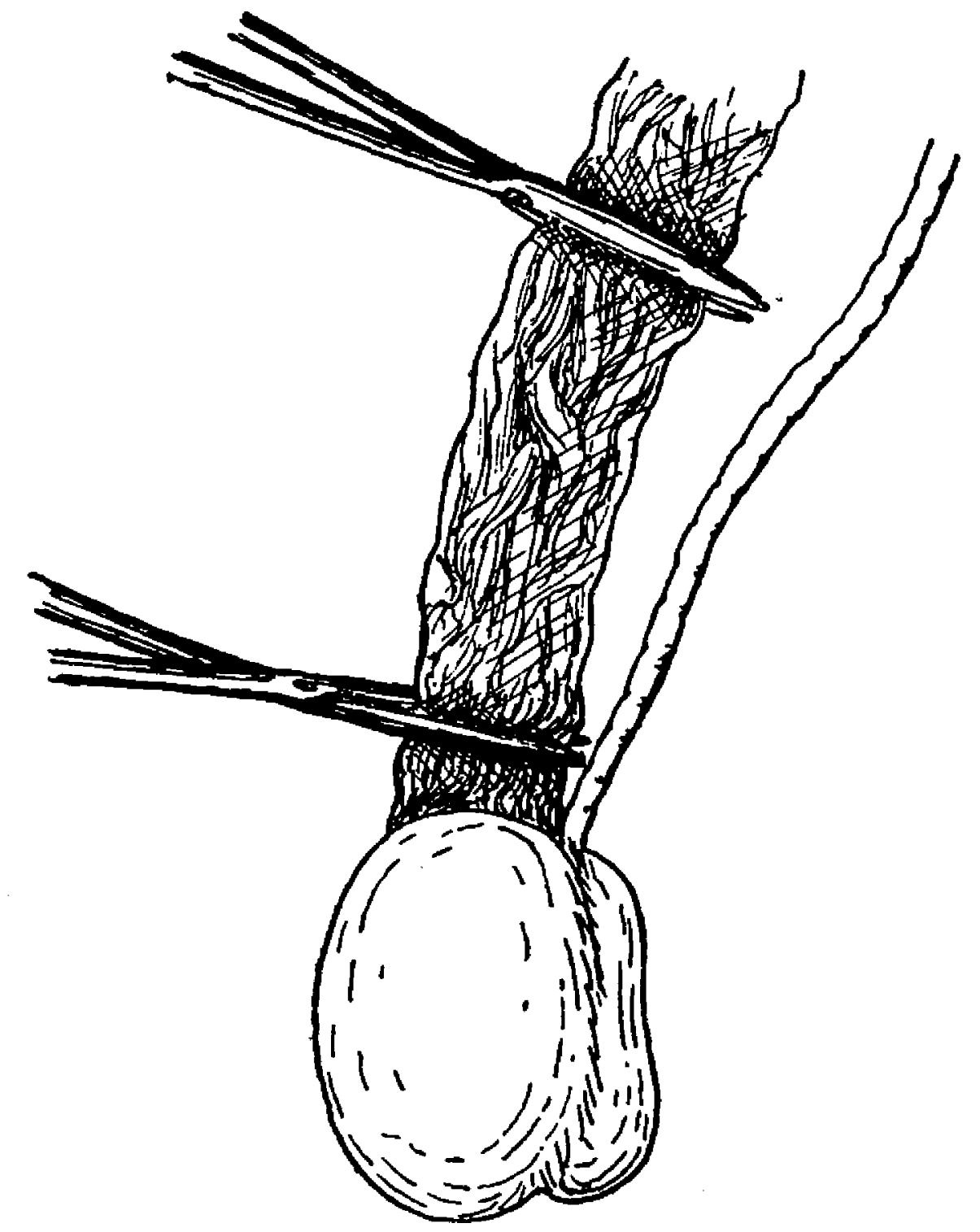

Fig. 1. 
top of the scrotum, and another close to the epididymis, and the whole mass cut away, leaving about a quarter of an inch projecting from each pair of forceps. (Fig. 1.) The two cut surfaces are now very carefully brought together by a continuous suture of the finest catgut (specially prepared so as to be reliably aseptic) passed with a very fine glover's needle. Commencing at one edge, the needle is passed through the entire thickness of the cut surfaces at very short intervals, until the other edge is reached and included; the suture is then brought back again in the same way to the starting-point, and the two ends firmly knotted together. The clamp forceps are now removed, and, if the sutures are properly applied, no bleeding takes place. (Fig. 2.) If, however, not quite dry, one or two points of interrupted suture will quite suffice

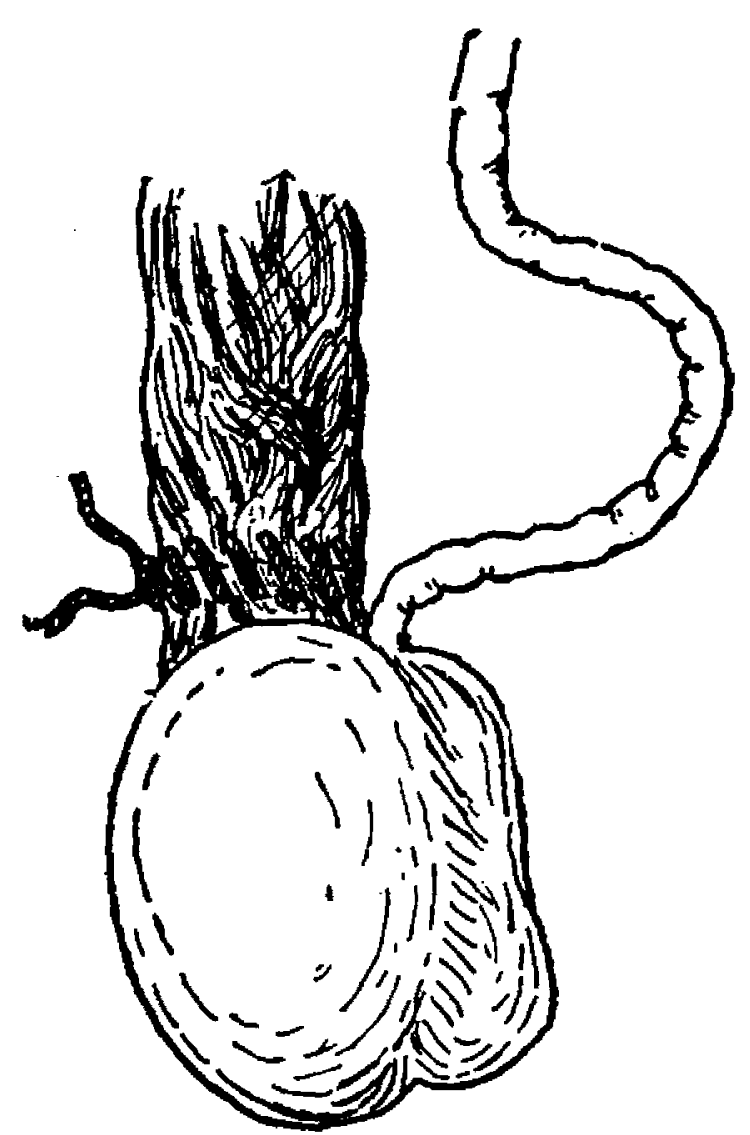

Fig. 2.--Varicocele Treated by Suture.

to completely staunch the wound. No ligature of any kind is employed. The scrotal incision is now closed by continuous catgut suture passed so as to include all the divided fascial coverings of the cord; no drainage is necessary. The afterpain is slight, and convalescence is usually complete in eight or ten days under a single antiseptic dressing. I have 
operated during the past five years on nine cases by this method; all of them were extensive varicoceles, the testicle hanging down 5 or 6 inches, and being much atrophied. All of them recovered without hæmorrhage, and none of them suppurated. Three have since passed into the Army Medical Service, and one has obtained a commission in the R.I.C.; in none has there been any recurrence.

Before adopting this method, where simply the veins were ligatured and excised, I found that the elongation of the scrotum continued after operation, and even was increased thereby; various methods have been recommended for bracing up the testis, but all of them are unsatisfactory. The suggestion of Mr. Bennett, to ligature the cord in two places and excise the mass in the usual way, and then knot the two ligatures together so as to shorten up the cord, appears to be unscientific, as it brings into apposition the two stumps rendered necrotic by the ligatures, so that adhesion cannot take place. By the method I have recommended, however, firm union takes place, and the cord is permanently shortened.

Another advantage is that no devitalised tissue is left to be absorbed and threaten the asepsis of the wound. The catgut used, however, must be above suspicion. I have found the best way to prepare it is as follows :-The finest dry gut is loosely coiled on a glass reel and put to soak for several days in a saturated solution of corrosive sublimate in ether; it is then taken out, the excess of solution shaken out, and kept for use in strong alcohol, which sufficiently reduces the strength of the corrosive sublimate. Only the very finest catgut should be used, as it is impossible completely to sterilise the interior of thick catgut. If for any purpose a stronger string is required, it can be easily prepared by twisting together several strands of the fine gut. I have tried experiments with gut prepared in this way and kept 
for a long time in alcohol, and invariably found it sterile in a cultivation medium.

A somewhat similar method of operation is eminently suitable for the excision of selected cases of nævus; in fact, it can be applied to almost any nærus which could be treated by ligature. Long glover's needles are passed under the nævus, ready threaded with boiled silk; each needle is entered a quarter of an inch from the margin of the nævus, under the growth, and out a quarter of an inch beyond the growth on the opposite side. The number of needles varies, of course, with the size of the nævus; they should be passed parallel to one another at distances of about half an inch apart, and extend from one extremity of the nævus to the other. All the needles are left in position without drawing the threads

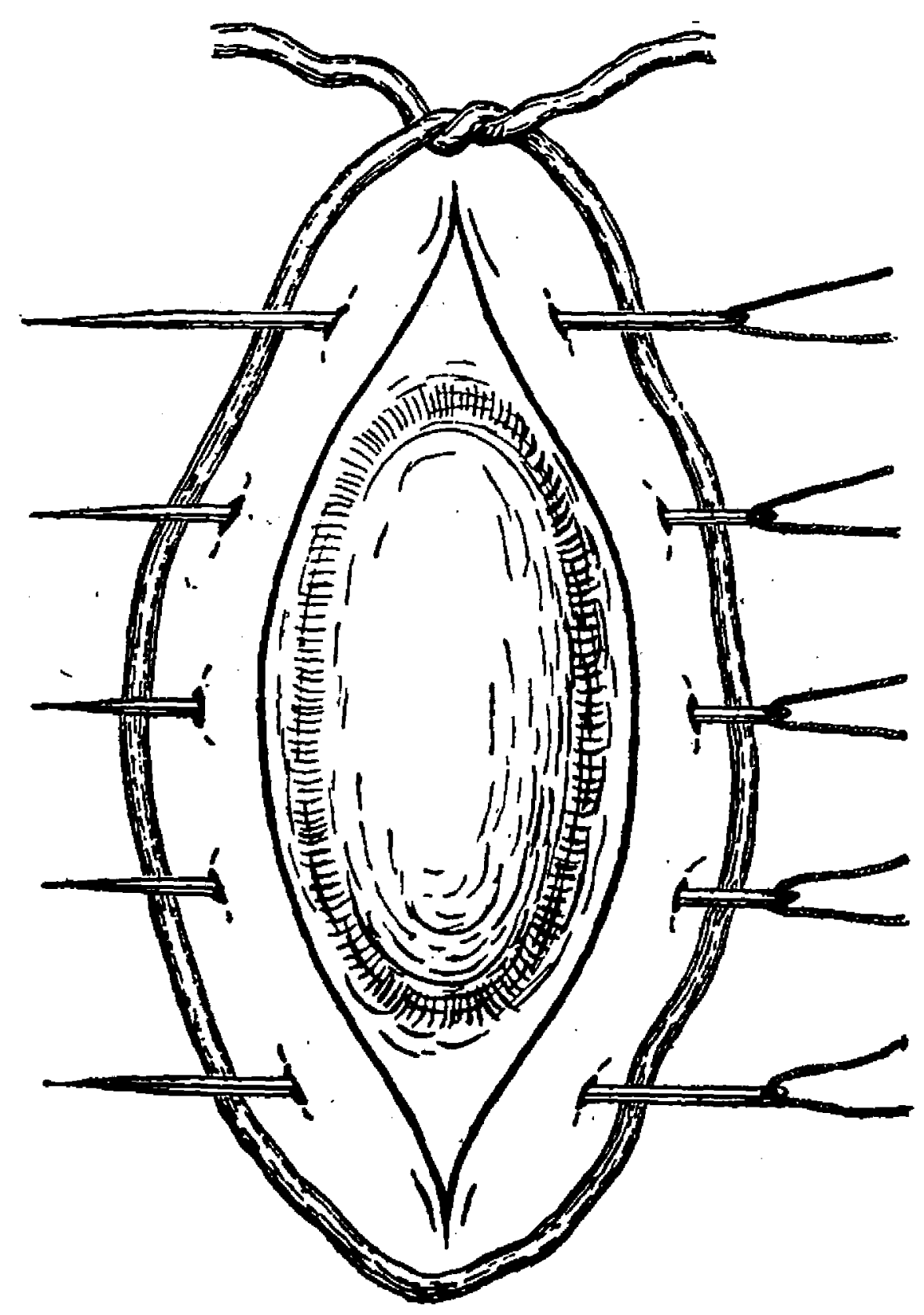

Fig. 3.-Nævus Treated by Suture. 
through, while an elastic ligature to temporarily arrest bleeding is provisionally tightened underneath the needles. The nævus can now be excised at leisure, as the bleeding is under control An elliptical incision is made down to the needles, leaving a margin of healthy skin all round, the elastic ligature is taken off, the silk threads rapidly pulled through, and the sutures firmly closed; if any bleeding comes from any point between the sutures, a few additional points of suture can be passed by a curved needle. (Fig. 3.) The advantages of obtaining a clean linear scar, healed in a week, over the slow sloughing away of a ligatured nævus are obvious.

I have operated in this way six times, and have every reason to be satisfied with the results.

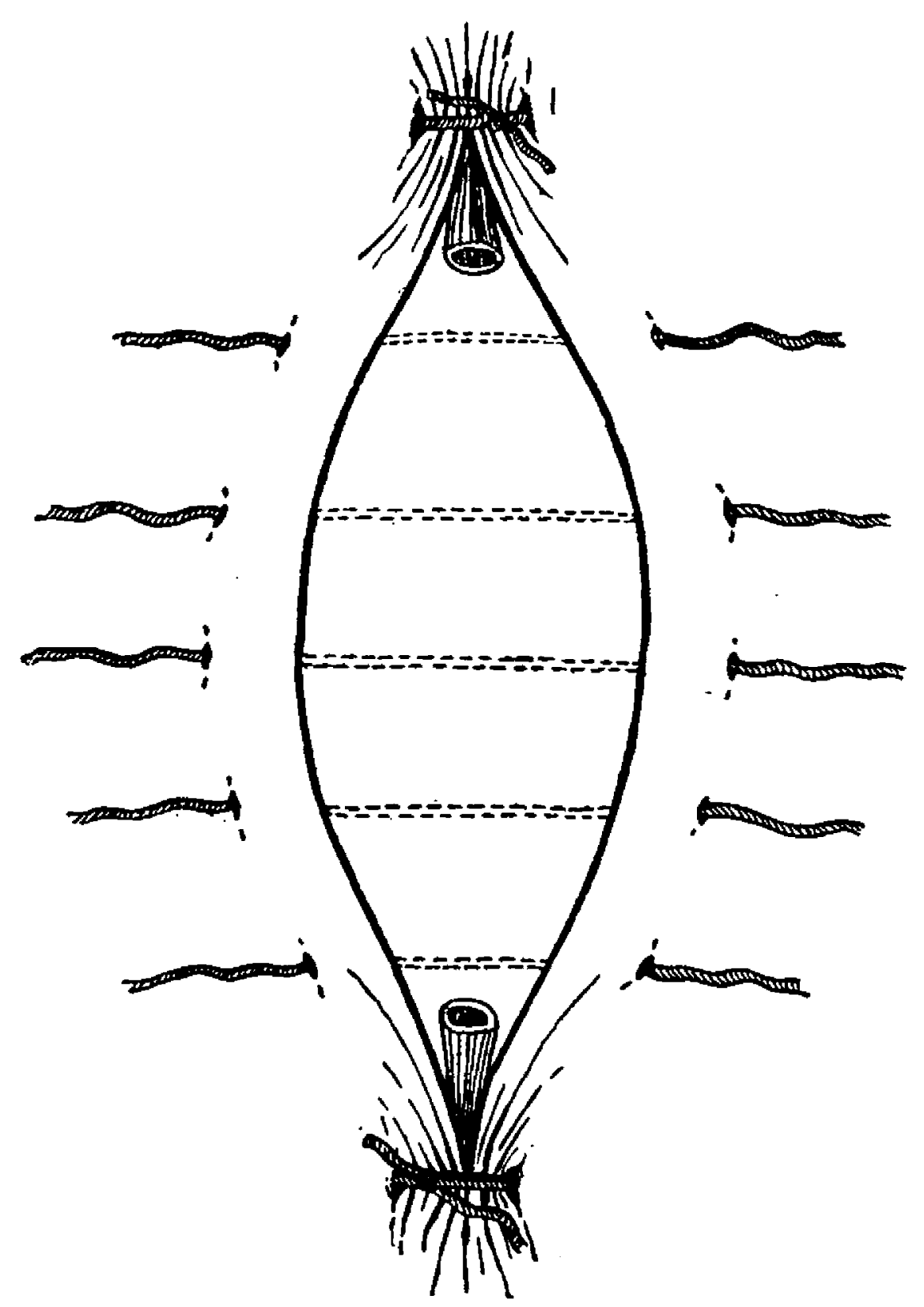

Fig. 4.-Varicose Veins of Leg Treated by Suture. 
In excision of varicose veins in the leg it assists much the aseptic healing if we dispense with ligatures to the divided veins. This can readily be done by means of sutures. An incision is made over the section of vein intended to be excised, the vein caught by two pairs of catch-forceps. divided between them, and dissected free to the upper and lower linits of the incision. A series of deep silk sutures are now passed under the wound, the one at each angle passing under the vein. These two sutures are first closed, and as they completely control the bleeding, the portions of vein to which the catch-forceps are attached may be snipped away close up to the sutures; a continuous fine catgut suture is applied to the edges of the wound, and, afterwards, the other deep sutures are closed so as to completely obliterate the wound cavity. (Fig. 4.) The deep sutures may be removed about the fifth day. I have operated eighteen times by this method with complete asepsis and satisfactory obliteration of the vein.

The above three operations illustrate what I consider to be useful expansions of the treatment of bleeding by sutures, and I am sure other developments of the method will occur to any surgeon who, from practical application of the procedure, becomes impressed with its utility.

The President thought that papers dealing with practical matters of detail of this sort were of more interest than the capital subjects of surgery. With regard to the question of the avoidance of ligatures, he agreed with Mr. Ball. Quite recently he had written a paper, published in the Dublin Journal of Medical Science, on operations on veins. He thought that sutures were better avoided in superficial operations which have to do with veins. Mr. Ball's passage of a ligature under the varicose vein is practically a soft ligature by acupressure. The President did not approve of the removal of large portions of veins. He would prefer several 
operations over a limited area. He has done hundreds of operations for the cure of varicose veins, and both he and his colleagues have given up large operations on veins. He agreed with nearly all Mr. Ball said on operations for varicocele. In that case pressure applied by a pad low down over the loose scrotum is not suitable. His own practice is to excise from half to one inch of the pampinniform plexus, pass a sterilised catgut ligature round the stump, and ligature the cut ends. He only tied the ligature round the stump tight enough to stop hæmorrhage. The part did not then necrose, and no slough was left. One great difficulty was the exact ligaturing of the skin of the scrotum, owing to the inversion of the skin, produced by the dartos muscle. He had learned a plan from the late Mr. Stapleton, of Jervis-street Hospital. It was called a post-mortem suture, and was passed from the deep surface to the skin. 'This produces eversion.

Mr. Bennetr bore testimony to the success of the cases mentioned by Mr. Ball. What he thought of most importance was his method of dealing with nævi. If the nævus were only the size of a shilling there was no necessity for cutting it out. Two ligatures could be passed at right angies, with an elastic ligature round them, and the little tumour contracted to nothing in a week. But in a large growing nævus in a young child what was to be feared was the loss of blood during the performance of the operation. By Mr. Ball's method no blood was lost. It was a much superior method to the different coloured strings adopted by Erichsen.

Dr. S. M. Thompson highly approved of deep sutures. Secondary hæmorrhage was not nearly so liable to occur, and the dressings could be left on for a considerable time.

Mr. Kendal Franks entirely agreed with what Mr. Bennett had said about nævus. He had never tried Mr. Ball's method, but hoped he would have an opportunity of trying it shortly. It was a new method. He did not think there was so much novelty in the other matters mentioned in this paper. He did not think there was yround for the terror which some people had for a drainage tube. Where there is considerable loss of substance beneath a wound, he generally preferred to insert a drainage tube after suturing the skin, and to apply pressure. In the majority of cases, however, he thought the deep suture the best. In breast operations, and above ull in amputations, he thought the deep suture the ideal method. He preferred the President's method of operating on varicocele to 
Mr. Ball's. With regard to operations on varicose veins, he did not see any difference between putting a ligature under the vein and tying it over the skin and tying it under the skin. It is quite unnecessary to remove large portions of reins. He thought that the success of operations on varicose veins depended on the after treatment. If the patient is allowed to walk about without any support to the limb, when the wound is healed, superticial veins will often enlarge. The patient must wear an elastic bandage or stocking for 4 to 6 months, until the collateral circulation through the deep veins is effected. As the deep veins lie between muscles, if they become varicose, it does not signify.

Mr. Thomson thought Mr. Ball's treatment of nævus was the most important practical suggestion. It seemed to him that the ligature had been put upon its trial. Mr. Ball had abandoned the term ligature for suture, but he maintained that Mr. Ball was only ligaturing the veins in another way. He thought his results were not due to this particular form of suture. Some of Mr. Ball's methods were neater methods, but not better in their absolute results.

Mr. TAYIor had seen a good many of the cases operated on by Mr. Ball. In the operation for varicocele, the catching of the veins by the forceps flattened them out, and so there was a good broad line of union on suturing. The shortening of the cord was important, because if the testicle was allowed to drop back into the scrotum it would draw on the cord and cause a recurrence of the pain, which is always present in cases of varicocele. He thought a very good way of closing the scrotal wound was to draw the angles of the wound apart, and put in a continuous suture. Since he had been to Vienna he was more than ever in favour of deep sutures.

Mr. Ball, replying, said that the President had used the terms "necrosis" and "slongh" as being practically synonymous terms. He did not agree with this. A necrotic part becomes a slough when it becomes septic. The more necrotic tissue, as ends of veins, that is present in a wound, the more likely is it to become septic. A certain amount of necrotic tissue can be absorbed, but the less there is in a wound the better. In varicocele, by suturing the cut veins together, there are no necrotic ends formed, as would have resulted had they been ligatured. In operating for varicose veins on the legs, he removed several small sections. In that operation the suture is like a ligature in that it surrounds the vessel. This, 
By Dr. C. B. Ball.

however, is not the case in the operations for nævus and varicocele. In the operation for varicose veins of the leg, he thought his method of introducing the ligature was easier, and certainly it was easier to take out. It also dispensed with two pieces of catgut lying inside, and any operative technique which enables a surgeon to dispense with dead matter in a wound is an advance. 